\title{
GEOSTATIONARY SATELLITE BASED RAINFALL ESTIMATION AND VALIDATION: A CASE STUDY OF JAVA ISLAND, INDONESIA
}

\author{
SUSENO, Dwi Prabowo Yuga ${ }^{1}$, Tomohito J. YAMADA 2 \\ ${ }^{1}$ Member of JSCE, M.Sc., River and Watershed Engineering Laboratory, Hokkaido University \\ (Kita 13, Nishi 8, Kita-ku, Sapporo, Hokkaido, 060-8628, Japan) \\ ${ }^{2}$ Member of JSCE, PhD, Associate Professor., River and Watershed Engineering Laboratory, Hokkaido University \\ (Kita 13, Nishi 8, Kita-ku, Sapporo, Hokkaido, 060-8628, Japan)
}

\begin{abstract}
Near real time rainfall information is necessary for early warning of rainfall triggered hazard such as floods and landslides. Remote sensing based rainfall estimation has been considered to be used to fulfill that purpose. This research is addressed to use geostationary based rainfall estimation by using Multi Transport Satellite (MTSAT) data which is blended with Tropical Rainfall Measuring Mission (TRMM) 2A12 datasets in order to provide near real time rainfall information, especially for hazard study purposes over Java Island, Indonesia. Comparison to TRMM Multi Precipitation Analysis (TMPA) datasets is performed. Spatial and temporal validation of those rainfall estimations is conducted by validating them with available rain gauge data during a rainy season in December 2007. Temporal validation result shows that TMPA demonstrated better statistical performance than MTSAT blended. However for the spatial correlation, MTSAT blended shows relatively better performance than TMPA.
\end{abstract}

Key Words : MTSAT, TRMM 2A12, TMPA, rainfall estimation, validation

\section{INTRODUCTION}

Floods and landslides are noticed as two of natural hazards that repeatedly occurred during rainy seasons in Indonesia. Floods (e.g.: inundation, flash flood, debris flow, etc) and landslide that triggered by severe storm have very serious impact to the loss both infrastructures and lives. As reported by SCTV (a private TV station in Indonesia) during December 2007, there were at least 21 of big floods events in 8 landslides events struck several different places in Java Island ${ }^{1)}$. Total casualties of the events for flood and landslide were 23 and 63 respectively and the events cause losses of thousand houses and infrastructures.

Many of the natural hazard casualties can be avoided if there is an early warning to the prone area due to the occurrence of successive high intensity of rainfall. The slow dissemination of measured rainfall information most likely is considered as serious obstacle in terms of the use of meteorological information for early warning purpose. It is because the limitation of meteorological infrastructure itself. Many of them are still using manual measuring and recording system instead of automatic measurement networks with telemetry system. Another drawback of rain gauges measurement is that they are limited in spatial coverage.

A different system of rainfall measurement that nearly real-time, covers wide area and depict spatial distribution of rainfall is necessary to implement. One of system that can be applied is satellite based rainfall estimation data products.

There are three methods that can use for estimating rainfall from the satellite based upon type of observation i.e.: (1) Visible and Infrared (VIS/IR) method; (2) Passive and Active Microwave Method (PMW); and (3) Multisensor Techniques Method ${ }^{2}$. The VIS/IR method uses indirect approach for rainfall estimation, i.e. according to top surface cloud characteristics such as shape, brightness, temperature etc. Since the sensor is carried out by geostationary satellite, the global coverage of rainfall estimation can be provided. The PMW method uses more direct rainfall estimation. It utilizes interaction between hydrometeor and microwave such as scattering and emission. The limitation of this method is that observations are currently only available from low earth orbit 
satellite. It makes lower temporal resolution of PMW observation than VIS/IR method. The mutisensor techniques combine the advantages of VIS/IR and PMW methods. The principle of multisensor techniques is to adjust the IR using the other dataset such as radar, rain gauge or other satellite datasets ${ }^{2}$. There are several ready to use satellite rainfall estimation products based on multisensor techniques such as TRMM Multi-satellite Precipitation Analysis (TMPA), Climate Prediction Morphing Method (CMORPH), Precipitation Estimation from Remotely Sensed Information using Artificial Neural Network Multispectral Analysis (PERSIANN-MSA), and Global Satellite Mapping of Precipitation $(\mathrm{GSMaP})^{2)}$. The datasets are mainly delivered via internet through their respective dedicated product web page location.

This research is addressed to perform rainfall estimation based on multisensor technique by blending Multi Transport Satellite (MTSAT) and Tropical Rainfall Measuring Mission (TRMM) 2A12 dataset in order to provide near real time rainfall information (hereafter referred to as MTSAT blended), especially for hazard study purposes. MTSAT that captured the hemisphere in a quite high temporal resolution (1 hour) has good capability to monitor atmospheric condition such as rainfall by using its VIS/IR sensors. However, cloud is opaque in VIS/IR spectral bands, so rainfall estimation by using those of spectral bands are mainly based on the cloud top characteristics. In order to get more accurate estimation, the advantage of TRMM is accommodated. TRMM has more direct rainfall estimation due to its capability to penetrate the cloud and interact with hydrometeor.

The method that is used in the MTSAT blended is mainly based on the algorithm developed by Maathuis et. $\mathrm{al}^{3)}$. The method has been chosen because it is relatively simple both in data need and process, affordable for non-meteorologist and low cost computing.

Although there are several ready in use rainfall estimations freely available, the development of rainfall estimation that uses fine resolution satellite such as geostationary satellite dataset is still quiet needed. The motivation of this study is to provide rainfall estimation for rainfall triggered hazard such as flash flood. Most of flash flood events occurred in small river basin with a drainage area of a few hundred square kilometers or less ${ }^{4}$. Moreover flash flood is triggered by very intense and short duration rainfall ${ }^{5}$. Some flash floods occurred in the tropical region such as Indonesia are triggered by isolated convective storm over small river basin. The current available satellite-based rainfall datasets that mainly cover global scale (i.e.: 0.25 degree latitude/longitude and 3 hours for TMPA) is considered relatively coarse resolution when it is used for flash flood early warning detection purposes. Another consideration is due to the indirect nature of the relationship between spectral information from satellite and corresponding rainfall that makes those of rainfall prediction area not universally applicable ${ }^{6}$.

There are two main objectives of this research. Firstly is to apply and modify the rainfall algorithm developed by Maathuis et. $\mathrm{al}^{3)}$. Secondly is to evaluate the performance of MTSAT blended and to compare with ready to use rainfall estimation TMPA dataset by validating both temporally and spatially with available rain gauge data.

\section{THE STUDY AREA AND DATASETS}

The study area is Java Island located on $5^{\circ} \mathrm{S}$ to $10^{\circ} \mathrm{S}$ and $95^{\circ} \mathrm{E}$ to $105^{\circ} \mathrm{E}$ (window size $5^{\circ} \mathrm{x} 10^{\circ}$ ). For the validation purpose due to limitation of time of study and the availability of measured rainfall data, we select only southern part of Central Java i.e. Yogyakarta city and its surrounding. The area of study and the validation area are presented in Figure 1 .

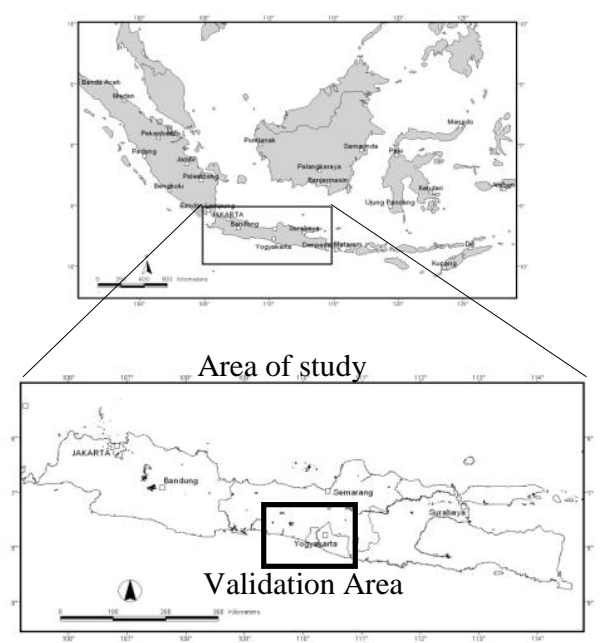

Fig.1 The study area of rainfall estimation and validation

The number of rainfall station situated in validation area is 22 automatic stations. Those of rainfall stations are operated by several different institutions i.e.: SABO Agency (Balai SABO) Yogyakarta, Public Work Agency of Progo Bogowonto Lukulo (Probolo Agency), Faculty of Geography, Gadjah Mada University and Agricultural Technology Research Agency (Balai Penelitian Teknologi Pertanian) of Yogyakarta.

The validation period is conducted during December 2007 (31 days). The raingauge data is 
mainly delivered in 1 hourly average rainfall, thus in this case we have 744 data. The same number of MTSAT images is acquired from WebGMSMTSAT/GMS (HIMAWARI) data processing on WWW, Earthquake Research Institute \& Institute of Industrial Science, University of Tokyo (http://webgms.iis.u-tokyo.ac.jp). TMPA datasets is derived from http://mirador.gsfc.nasa.gov.

\section{METHOD AND VALIDATION SCHEMES}

\section{(1) The blending process}

The original method described by Maathuis et.al. ${ }^{3)}$ uses Meteosat Second Generation (MSG) and TRMM as data input. Because this study is conducted over Java Island that isn't covered by MSG, so we use MTSAT and TRMM as data input. We assume that the IR band $(10.8 \mu \mathrm{m})$ of MSG is comparable to IR1 $(10.3 \mu \mathrm{m}-11.3 \mu \mathrm{m})$ of MTSAT and the water vapor band $(6.2 \mu \mathrm{m})$ of MSG is comparable to IR3 $(6.5 \mu \mathrm{m}-7 \mu \mathrm{m})$ of MTSAT.

The basic idea of MTSAT blended is how to develop statistical relationship between cloud top temperature depicted by MTSAT IR1 datasets and rain rate estimated by TRMM 2A12 datasets. For the convective cloud situation, the relationship between cloud top temperature and rain rate shows that the low cloud top temperature is associated with heavier rainfall ${ }^{7)}$. A statistical regression should be developed to express that relationship. We use exponential curve to draw such statistical relationship. It means that the rain rate is decreasing exponentially along the increasing of cloud top brightness temperature ${ }^{8}$. The developed statistical regression will be used to generate rainfall estimation based on MTSAT datasets.

The most important step during performing this method is how to get collocated image both temporally and spatially between MTSAT and TRMM dataset. It is a prerequisite in order to develop strong statistical relationship between cloud top temperature and rainfall rate. The ideal collocated image is those of MTSAT data and TRMM data that have the same acquisition time over the same area. In the real situation this ideal condition is difficult to fulfill. We can only select collocated image that have almost the same time of acquisition over the area. Because of the lag of acquisition time, a slightly discrepancy of cloud spatial distribution is depicted in collocated image.

In order to reduce such discrepancy, an averaging process for TRMM data based on the grouped MTSAT-IR cloud temperature (e.g.: $0.5 \mathrm{~K}$ or $1 \mathrm{~K}$ equal range temperature) is performed ${ }^{3)}$. This process can increase the correlation coefficient of the relationship between cloud top temperature and rainfall rate.

We also try to reduce such discrepancy by limiting the coverage of collocated image during statistical relationship development. This process is performed to improve the original algorithm that used whole coverage as collocation window. We adopted the collocated window size that used by Heinemann et. al. ${ }^{9)}$ i.e.: $5^{\circ} \times 5^{\circ}$ latitude/longitude to divide the whole domain window size into two smaller windows. We assumed that the limiting window size could estimate spatial collocation of cloud distributions between MTSAT and TRMM. We examine the relationship of both windows by comparing the correlation coefficient. The best statistical relationship is chosen to estimate the rainfall of whole coverage area.

The last process for rainfall data generation is performed by the best regression equation based on MTSAT cloud temperature. The rainfall data generation process is only applied to the MTSAT cloud top temperature that considered as potential precipitating cloud. The potential precipitating cloud has been selected by the brightness temperature difference between IR1 and IR3 that is less than $11 \mathrm{~K}^{3}$. Each regression function will valid to certain range of MTSAT image series. Figure 2 shows the schematic diagram of MTSAT blended in this study.

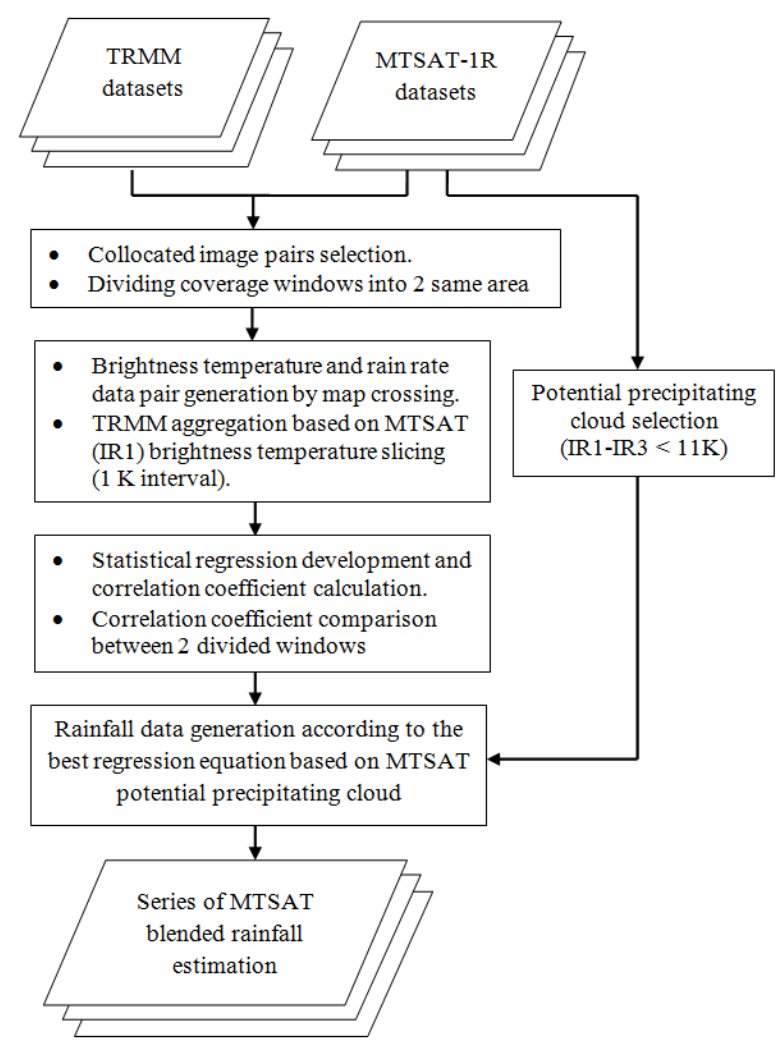

Fig.2 Blending method between MTSAT and TRMM data in this study 


\section{(2) Validation Schemes and Statistical Analysis}

In order to measure the performance of MTSAT blended rainfall estimation, we validate by comparing it with rain gauge data both in temporally and spatially scales. As a comparison, we also perform the same validation process to TMPA datasets.

Temporal validation is performed in point to pixel basis i.e.: point rainfall data from rain gauge measurement and pixel based rainfall estimation from satellite. Hourly average and 3 hourly average rain gauge data are used to validate MTSAT blended and TMPA rainfall estimation respectively. Pixel information that contains rainfall estimation from satellite is retrieved according to the coordinate location of rainfall stations. For each rain gauge location, a pair of estimated and observed rainfall has been generated.

Statistical comparison has been performed for those of pair data. We use some categorical statistics such as accuracy, bias score, Probability of Detection (POD), False Alarm Ratio (FAR) and Critical Success Index (CSI) to evaluate the performance of rainfall estimation from satellites.

A dichotomous method is used to say 'yes' if rain $\neq 0$ and to say 'no' if rain $=0$. There are four combinations possible when we compare between yes/no of observed and yes/no of estimation (see Table 1).

- Hit - if both estimation and observed say 'yes'

- Miss - if estimation said 'no' but observed say 'yes'

- False alarm - if estimation said 'yes' but observed say 'no'

- Correct negative - if both estimation and observed say 'no'

Table 1 Contingency table to summarize the number of hits, false alarms, misses and correct negative that is used to calculate categorical statistics.

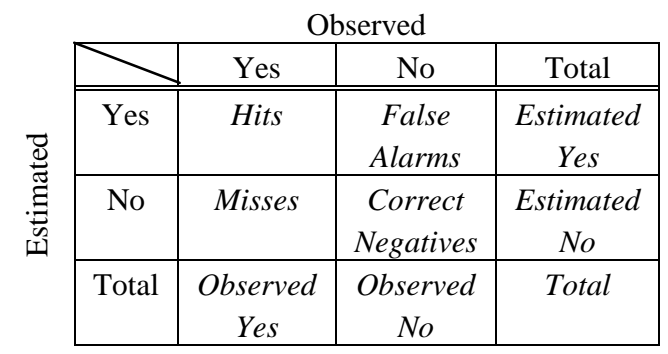

The categorical statistic parameters are calculated according to the following equations ${ }^{10)}$ :

$$
\begin{gathered}
\text { Accuracy }=\frac{\text { hits }+ \text { correct negatives }}{\text { total }} \\
\text { Bias score }=\frac{\text { hits }+ \text { false alarms }}{\text { hits }+ \text { misses }}
\end{gathered}
$$

$$
\begin{gathered}
P O D=\frac{\text { hits }}{\text { hits }+ \text { misses }} \\
F A R=\frac{\text { false alarms }}{\text { hits }+ \text { false alarms }} \\
C S I=\frac{\text { hits }}{\text { hits }+ \text { misses }+ \text { false alarms }}
\end{gathered}
$$

Spatial validation is performed by calculating spatial correlation both in pixel to point and pixel to pixel basis. Spatial correlation is defined as the correlation between estimated and observed rainfall with respect to their geographical locations. In this study we only investigate the spatial correlation of convective rainfall cases. For pixel to point validation, the spatial correlation between rainfall estimation from satellite and corresponding measured rainfall data is calculated. In terms of pixel to point validation there is a remaining problem which is related to the type of data itself. Rainfall observation is considered as point data type otherwise satellite based precipitation is area data type (measured as pixel area) ${ }^{11)}$. In order to make comparable validation, a pixel to pixel based spatial correlation is conducted. It means that the pixel from satellite rainfall estimation is compared with the pixel (grid) of interpolated observed rainfall which has the same geo-reference. The interpolated observed rainfall is generated by using the block kriging interpolation method.

\section{RESULTS}

\section{(1) Temporal validation}

During the validation period, we have identified 36 collocated images. Some of them have 2 collocations in one day. The effect of limiting the coverage of collocated image is explained below. We calculate correlation coefficient $\left(r^{2}\right)$ of whole window coverage and those of two divided windows. We select the best $r^{2}$ between two divided windows and plot it against $r^{2}$ derived from whole window. For all collocation events the plotting result is shown in Figure 3.

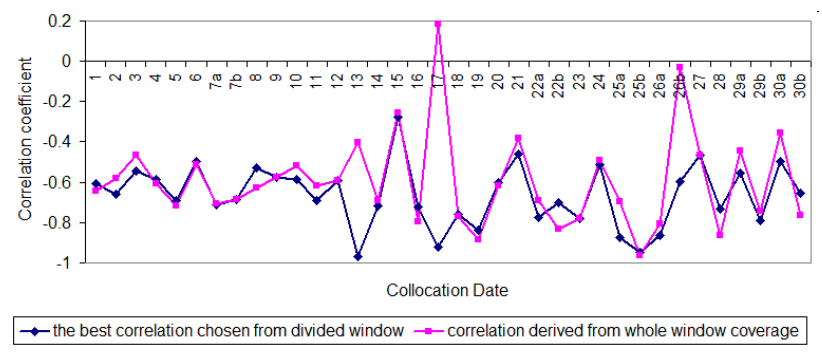

Fig.3 Comparison of correlation coefficient of the collocated images. 
According to Figure 3, it can be noticed that limiting window coverage can significantly improve the statistical relationship. It is indicated by increasing $\mathrm{r}^{2}$, especially in the collocation date 13 , 17 and 26b. The discrepancies that are identified in those of collocation times mainly are the rainfall which is still detected in TRMM but actually have dissipated in MTSAT image and/or vice versa. This result shows that the limiting window process is proven useful to reduce discrepancy by separating and rejecting it in one window and choose the other one that has less discrepancy.

In relation to temporal validation, the result is shown in Table 2. It can be examined that TMPA has higher accuracy than MTSAT blended. The result indicates that $72 \%$ of TMPA estimation is correct while MTSAT blended is only $59 \%$ correct. This condition is likely related to the probability of good collocation images that may be occurred during rainfall estimation process. TMPA has higher probability to have good collocation because it uses several PMW data compared with MTSAT blended that only use one PMW data.

According to bias score, it shows that MTSAT blended have a tendency to be overestimated and TMPA tend to be slightly underestimated. It indicates that potential precipitating cloud is more frequently detected in MTSAT blended than TMPA. This situation is consistent with high POD value. The FAR value of MTSAT blended is also quite high and it is indicated that many potential precipitating cloud detected by MTSAT images which are not producing rain. The CSIs of both MTSAT blended and TMPA are quite low i.e. only $20 \%$ and $38 \%$ (respectively) of 'rain' event both observed and/or estimated are correct. Based on those of statistical scores, it concludes that TMPA has better performance than MTSAT blended in terms of temporal validation.

Table 2 Summary of categorical statistic for MTSAT blended and TMPA.

\begin{tabular}{|l|c|c|}
\hline $\begin{array}{c}\text { Categorical statistic } \\
\text { parameters }\end{array}$ & $\begin{array}{c}\text { MTSAT } \\
\text { blended }\end{array}$ & TMPA \\
\hline \hline Accuracy & 0.59 & 0.72 \\
bias score & 4.54 & 0.78 \\
POD & 0.83 & 0.49 \\
FAR & 0.79 & 0.33 \\
CSI & 0.20 & 0.38 \\
\hline
\end{tabular}

\section{(2) Spatial validation}

Two convective storms have been chosen as the case study for spatial validation. The first case is 16 December 2007 storm that consist of series of MTSAT images from 06:30 UTC - 15:30 UTC. The second is 18 December 2007 from 04:30 UTC 12:30 UTC.

For the comparison purpose, series of TMPA data is also selected for the same time range of storm event. Otherwise, we cannot perform pixel to point validation such as performed in MTSAT blended spatial validation, due to the large size of TMPA's spatial resolution $\left(0.25^{\circ} \times 0.25^{\circ}\right)$. In this case we compare TMPA rainfall estimation with the group of rainfall station as average observation rather than individual. The group of rainfall stations is defined according to the spatial resolution of TMPA. The centroid of corresponding TMPA's pixel has been considered as coordinate location of each group. Spatial correlation is calculated based on those average rainfall observation from group of stations and TMPA rainfall estimation, in the same coordinate location.

Based on those two convective storm cases, spatial correlations of both pixel to point and pixel to pixel are conducted. The result is presented in Table 3.

Table 3 Comparison of spatial correlation between MTSAT and TMPA for two convective rainfall cases in pixel to point and pixel to pixel basis.

\begin{tabular}{|l|c|c|}
\hline & Pixel to point & Pixel to pixel \\
\hline \hline \multicolumn{3}{|l|}{ MTSAT blended } \\
\hline 16 December 2007 case & 0.36 & $0.50^{*}$ \\
\hline 18 December 2007 case & 0.33 & $0.61^{*}$ \\
\hline TMPA & 0.56 \\
\hline 16 December 2007 case & 0.46 & 0.36 \\
\hline 18 December 2007 case & -0.1 & \\
\hline
\end{tabular}

$*=$ Correlation is significant at the 0.05 level (2-tailed).

Firstly, we compare pixel to point spatial correlation of MTSAT blended and TMPA for both 16 December 2007 and 18 December 2007 convective storms. The MTSAT blended spatial correlations for both storm cases are 0.36 and 0.33 respectively. Otherwise, the TMPA rainfall estimation gives the result 0.46 and -0.1 respectively. The low correlation can be readily explained due to spatial offset between cloud producing rain pixel and location of rainfall stations. Positive correlation indicated that spatial variation of the observed rainfall can be depicted by satellite image, conversely negative correlation shows that the observed rainfall distribution is not well represent by satellite image.

Those of results demonstrate that MTSAT blended can well represent the convective storms in both cases. Otherwise, TMPA can only represent convective storm distribution for the first case and failed to represent the second case. 
Secondly, we compare pixel to pixel spatial correlation to the same convective storm cases. In order to make comparable results between MTSAT blended and TMPA validation, we perform the block kriging interpolation and use the same block size as TMPA's spatial resolution. The result is confirmed that after spatial interpolation, observed rainfall can be well represented by both MTSAT blended and TMPA rainfall estimation. We calculate the average of spatial correlation of MTSAT blended and TMPA from both two cases. We get the result of 0.56 and 0.46 for MTSAT blended and TMPA respectively. It shows that in average MTSAT blended demonstrates better spatial representation of convective rainfall than TMPA.

The average value of spatial correlation of MTSAT (i.e.: 0.56) has good agreement with the result of Ebert and Manton's study ${ }^{12}$ ) that for the instantaneous rainfall of mixed geostationary satellite and polar orbit satellite (IR-SSM/I) algorithms has correlation coefficient ranging from 0.49 to 0.55 .

\section{DISCUSSION AND CONCLUSIONS}

Regarding to the temporal validation, TMPA shows relatively better performance than MTSAT blended. It could be explained mainly because of TMPA algorithm developed based on various data inputs as well as complex algorithm. The relatively low statistical performance of MTSAT blended rainfall estimation compared to TMPA is considered as the limitation of this method. It might be because of the overestimate of potential precipitating cloud detected by MTSAT blended. For the further research, the more accurately potential precipitating cloud detection should be adopted. Furthermore, limiting collocated window method that already shows a promising result should be enhanced.

However for the spatial correlation, MTSAT blended is demonstrated better performance than TMPA. With regards to this potentiality, MTSAT blended algorithm is quite promising as an alternative method for providing rainfall data for ungauge remote area. It can be used also as an indirect approach for the rainfall triggered hazard early warning system. It is because MTSAT blended has shown quite good to represent spatial distribution of rainfall especially convective storm events, though the accuracy of rainfall detection should be increased. The convective storm that characterized by very intense rain in short duration (lasting less than 1 hour) and covers small area ${ }^{5}$. This type of rainfall has been considered as the causing factor of flash flood6). Based on this relation, MTSAT dataset that have good spatial and temporal resolution can be considered to be used for such kind of rainfall related hazard studies. Another consideration is that MTSAT blended is only need simple data input and processing and low cost computing. This consideration is beneficial in term of practical point of view.

ACKNOWLEDGMENT: Thanks to Dr. Ben Maathuis, Dr. Projo Danudoro, M.Sc and Dr. Pramono Hadi, M.Sc for their valuable critical comments and suggestions during the research. Thanks are also due to the anonymous reviewer whose comments significantly improved the quality of this paper.

\section{REFERENCES}

1) SCTV: Liputan 6, from www.liputan6.com, 2008.

2) Kidd, C., V. Levizzani.: Status of satellite precipitation retrievals, Hydrol. Earth Syst. Sci. Discuss 7, pp.8157-8177, 2010.

3) Maathuis, B. H. P., A.S.M Gieske, V. Resios, B.V. Leeuwen, C.M. Mannaerts, J.H.M Hendrikse.: Meteosat-8 From temperature to rainfall, ISPRS Commission VII Mid-term symposium Remote sensing: from pixel to processes, Enschede, The Netherlands, 2006.

4) National Research Council: Flash Flood Forecasting Over Complex Terrain With an Assessment of the Sulphur Mountain NEXRAD in Southern California, The National Academies Press, Washington DC, 2005.

5) Dingman, L. S.: Physical Hydrology, New Jersey, Prentice Hall, 2002.

6) Wardah, T., S. H. Abubakar, A. Bardossy, M. Maznorizan: Use of geostationary meteorological satellite images in convective rain estimation for flash-flood forecasting, Journal of Hydrology., Vol.356,pp.283-298, 2008.

7) Kuligowski, R.: Remote Sensing in Hydrology, From: http://www.weather.gov/iao/InternationalHydrologyCourse CD1/1029/wmo_bk.ppt, Retrieved: 4 November 2007.

8) Vicente, G. A., R.A. Scofield, W. P. Menzel.: The Operational GOES Infrared Rainfall Estimation Technique, Bulletin of the American Meteorological Society, Vol.79(9), pp.1883 - 1898, 1998.

9) Heinemann, T., A. Lattanzio, F. Roveda.: The Eumetsat Multi-sensor Precipitation Estimate (MPE), From: http://www.eumetsat.int/groups/ops/documents/document/m pe_introduction_ipwg_2002.pdf, Retrieved: 20 April 2008.

10) Ebert, E. E.: Forecast Verification-Issues, Methods and FAQ.From:http://www.bom.gov.au/bmrc/wefor/staff/eee/ve rif/verif_web_page.html, Retrieved: 28 August, 2007.

11) Grimes, D. I. F., E. Pardo-Iguzquiza, R. Bonifacio.: Optimal areal rainfall estimation using raingauges and satellite data, Journal of Hydrology Vol.222,pp.93-108, 1999.

12) Ebert, E. E., Michael J. Manton.: Performance of Satellite Rainfall Estimation Algorithm during TOGA COARE, J. Atmos. Sci Vol.55,pp.1537 - 1557, 1998.

(Received September 30, 2010) 